\title{
MULTIPLE FUNCTIONS OF INOSITOLPHOSPHORYLCERAMIDES IN THE FORMATION AND INTRACELLULAR TRANSPORT OF GLYCOSYLPHOSPHATIDYLINOSITOL ANCHORED PROTEINS IN YEAST
}

\author{
Régine Bosson and Andreas Conzelmann \\ Department of Medicine/Biochemistry, University of Fribourg, Switzerland
}

\begin{abstract}
Corresponding author e-mail: andreas.conzelmann@unifr.ch
Dr. A. Conzelmann, Department of Medicine, Division of Biochemistry, Chemin du Musée 5, CH1700 Fribourg, Switzerland. Tel. ++4126300 8630. Fax ++41263009735.
\end{abstract}

Running title: GPI biosynthesis in yeast

Key words: Glycosylphosphatidylinositol, Saccharomyces cerevisiae, biosynthesis, cell wall, ceramide, lipid remodeling 


\begin{abstract}
The mature sphingolipids of yeast consist of inositolphosphorylceramides (IPCs) and glycosylated derivatives thereof. Beyond being an abundant membrane constituent in the organelles of the secretory pathway, IPCs are also used to constitute the lipid moiety of the majority of GPI proteins, while a minority of GPI proteins contain Phosphatidylinositol (PI). Thus, all GPI anchor lipids (as well as free IPCs) typically contain C26 fatty acids. However, the primary GPI lipid that is added to newly synthesized proteins in the ER consists of a PI with conventional C16 and C18 fatty acids. A new class of enzymes is required to replace the fatty acid in sn-2 by a C26 fatty acid. Cells lacking this activity make normal amounts of GPI proteins but accumulate GPI anchors containing lyso-PI. As a consequence, the ER to Golgi transport of the GPI protein Gas1p is slow and mature Gas1p is lost from the plasma membrane into the medium. The GPI anchor containing C26 in sn-2 can further be remodeled by the exchange of diacylglycerol for ceramide. This process is also dependent on the presence of specific phosphorylethanolamine side chains on the GPI anchor.
\end{abstract}

Abbreviations: CWP = cell wall protein; DAG = diacylglycerol; GlcN, GlcNH $\mathrm{H}_{2}=$ D-glucosamine; GPI = glycosylphosphatidylinositol; IPC = inositolphosphorylceramide; Man = mannose; PI = phosphatidylinositol; PMP = plasma membrane protein.

\title{
1. INTRODUCTION
}

GPI anchoring is utilized by most eukaryotes to express proteins at the cell surface and Saccharomyces cerevisiae does not make an exception to this. Glycosylphosphatidylinositol (GPI) anchors are structurally complex glycophospholipids, which are added posttranslationally to the Cterminal end of secretory proteins after they have been translocated into the ER. While a few yeast 
GPI proteins reside permanently at the plasma membrane, a majority of them gets further processed and is integrated into the cell wall by a covalent attachment to cell wall glucans. Some of the estimated 60 GPI proteins predicted from the yeast genome sequence serve enzymatic functions required for the biosynthesis and the continuous shape adaptations of the cell wall, others seem to be structural elements of the cell wall, yet others mediate cell adhesion, and a few seem to have other functions [1-4]. The GPI biosynthetic pathway is absolutely necessary for growth and survival of yeast cells [5].

In all organisms, the precursors of GPI anchored proteins have a classical signal sequence for import into the ER at their N-terminus and a GPI anchoring signal at their C-terminus; the Cterminal signal is necessary and sufficient to direct GPI addition [6]. The C-terminal GPI anchoring signal is recognized and removed by a GPI transamidase, which replaces it by the preformed GPI. GPI anchoring signals are composed of a C-terminal hydrophobic domain, which is separated by a short hydrophilic spacer from the cleavage/attachment site ( $\omega$-site) [7-9]. Fig. 1A shows the average structure of the yeast GPI anchor as elaborated from the total pool of GPI anchors extracted from yeast cells without the prior purification of any particular GPI protein $[10,11]$. The structure of the GPI core linking the protein to the lipid moiety is highly conserved among all eukaryotes, as is true for the enzymes that elaborate the GPI structure. Amazingly, even certain side chains such as the forth mannose (Man4) or the phosphorylethanolamine side chains added on Man1 and Man2 (Fig. 1A) are present in both, man and yeast and thus may have been conserved across long evolutionary distances. Clearly, the GPI biosynthesis pathway is a very ancient and highly conserved pathway.

Twenty years after the complete structures for the variant surface glycoproteins of Trypanosoma brucei and the mammalian Thy-1 glycoprotein had been worked out by the pioneering work of Mike Ferguson, Steve Homans and their coworkers in 1988 [12, 13], genes required for the addition of about everyone of the different structural elements of the GPI anchor have been 
identified, but new subunits and regulatory elements of the identified enzymes still continue to be discovered. In yeast the GPI lipids are synthesized in the ER and added onto proteins by a pathway comprising 12 steps, carried out by 23 gene products, 19 of which are essential. Excellent recent reviews summarize current knowledge about the biosynthesis of GPI anchors and the enzymes that are necessary for their elaboration [14-19].

The present review focuses on our current understanding of the exchange of the lipid moiety of GPI anchors in yeast i.e. on GPI lipid remodeling, a phenomenon that is required to introduce ceramides into the GPI anchor and seems to be paradigmatic for a number of protozoa and fungi.

\section{BIOSYNTHESIS OF GPI LIPIDS IN YEAST}

The genes encoding the different enzymes involved in the biosynthesis of GPI anchors have been cloned mostly by the transfection of gene libraries into mammalian or yeast gpi mutants, which are deficient in GPI anchoring and therefore retain all GPI proteins in the ER so that they have no GPI proteins at their surface. By selecting for gpi transformants that reexpressed GPI proteins at their surface and thus contained a gene able to complement the mutant phenotype, a great number of genes have been identified, foremost by the group of Taroh Kinoshita in Japan and also by several other groups. The various steps are the same in yeast and in mammals and the genes involved in each step are summarized in Fig. 2. All genes indicated in Fig. 2 are essential except for GPI1, ERI1, GPI7 and BST1. This list of genes may still be incomplete and one can speculate that there would also be a need for a flippase flipping PI-GlcN towards the ER lumen (Fig. 2). Moreover, flippases for other substrates such as acyl-CoA, dolichylphosphorylmannose and phosphatidylethanolamine may equally be involved. Although for all steps some subunit has been shown to be, or can safely be assumed to be the catalytic subunit, there remains a lot to be learned about the exact roles of additional subunits, which are required at steps 1, 4, 9, 10 and 11 of Fig. 2. 
Another riddle to be solved concerns the role of the fatty acid that is added to the inositol of GPI lipids in many organisms, but which is usually taken off again just before or soon after the GPI lipid is added to proteins (steps 3 and 12, Fig. 2).

\section{REMODELING OF GPI LIPID MOIETIES}

\subsection{General considerations}

Lipid remodeling is invoked in instances, in which the lipid moiety of the free GPI lipid or the GPI anchor attached to a protein is modified. One can infer a remodeling event if an early intermediate of the GPI lipid biosynthetic pathway, e.g. GlcN-acyl-PI, carries a different lipid moiety than later intermediates or protein-bound GPI anchors. Practically however, it often is not trivial to obtain sufficient material of the earliest GPI intermediates for analysis. In some cases there are significant amounts of GlcN-acyl-PI, but the bulk of this material may not be destined for GPI anchor biosynthesis, but rather serve to generate free GPI lipids. This seems to be the case for many mammalian cell lines that produce much more GPI lipids than what is needed for protein anchoring [20-23]. In these cases it needs to be demonstrated that an early intermediate really is in the pathway leading up to protein-anchoring GPI structures. In yeast, free GPI lipids are present in so low amounts that they are not easily detected and all information on GPI lipids derives from mutant cells having mutations in the GPI biosynthetic pathway.

\subsection{GPI remodeling in protozoa}

\section{Trypanosoma brucei}

Historically, Trypanosoma brucei, the African sleeping sickness causing agent, was the first organism, in which GPI lipid remodeling was demonstrated. The GPI anchors of variable surface protein (VSG) of Trypanosoma brucei and Trypanosoma congolense are unique in having 
exclusively myristate (C14:0) as fatty acid component of their diacylglycerol (DAG) moiety. While VSG is expressed by the blood forms present in the mammalian host, the procyclic froms of T. brucei express other GPI proteins, which contain different GPI anchor lipids. The myristates of VSG are introduced into GPI lipids by two independent and distinct pathways. First, the free GPI lipid undergoes "fatty acid remodeling" to replace both its fatty acids with myristate during the final phase of GPI biosynthesis [24]. Second, the myristoylated anchor undergoes a "myristate exchange" reaction, leading to the replacement of myristate by another myristate in both sn-1 and sn-2. This second reaction occurs mainly on the GPIs that already are linked to VSG. The fatty acid remodeling pathway and the myristate exchange are distinct as they have different enzymatic properties and probably occur in different subcellular compartments, but both pathways require myristoyl-CoA as a substrate [25]. The exchange reaction is proposed to be a proofreading system to ensure that the fatty acids on VSG are exclusively myristate [25, 26].

The starting substrate for the first remodeling reactions in the bloodstream forms are complete GPI lipids containing C18:0 in sn-1 and C18:0, C18:1, C18:2, C20:4 or C22:6 in sn-2 [27]. The first reaction occurs at the sn-2 position of glycerol, and is followed by the subsequent attachment of myristate, donated from myristoyl-CoA. Then, the stearic acid at sn-1 is removed and the second myristate is incorporated. Monoacylated GPI (lyso-GPI) intermediates can be isolated in both cases [28]. An acyl-CoA binding protein (ACBP) showing a high affinity for myristoyl-CoA and enhancing fatty acid remodeling of GPI precursors in the trypanosome cell-free system has been cloned [29].

\section{Trypanosoma cruzi}

Trypanosoma cruzi is the causative agent of Chagas' disease. Most of the surface proteins and glycoconjugates are attached to the membrane by a GPI anchor. The major free glycosylinositol phospholipids (GIPLs) and protein-linked GPIs in T. cruzi contain ceramide as lipid moiety. An alkylglycerol, either as a lyso species or acylated, has also been observed in GPI anchors whereas 
DAG has never been detected. A recent study shows that the first step of the GPI biosynthesis does not use IPC as a substrate. This suggests that $T$. cruzi exchange a DAG for a ceramide generating a GPI anchor with a ceramide-based lipid, as it is the case in yeast [30].

\section{Aspergillus fumigatus}

Aspergillus fumigatus is a filamentous fungus and human pathogen that expresses GPI-anchored proteins on its cell surface. Isolation of four GPI-anchored proteins and analysis of their anchor revealed that the lipid part consists exclusively of IPC, most likely containing C18phytosphingosine amide-linked to a monohydroxylated C24 saturated fatty acid. In contrast to yeast, no DAG was found in the GPI anchors of A. fumigatus [31]. A galactomannan polysaccharide of $A$. fumigatus has been shown to be GPI-anchored and to contain the same ceramide as the one present in GPI-anchored proteins [32]. A. fumigatus, as many other fungi, also contains glycosylinositol phosphorylceramides, which consist of a similar, ceramide-based GPIlike structure [33]. As in other organisms, a cell free assay system shows that $A$. fumigatus initiates GPI biosynthesis by the addition of GlcNAc to PI to give GlcNAc-PI (step 1, Fig. 2)[34]. This suggests that a lipid exchange must occur to replace the base-sensitive diacyl lipid moiety of the early GPI intermediate by a ceramide.

\section{Leishmania mexicana}

Leishmania mexicana are parasites alternating between a flagellated promastigote stage that proliferates in the midgut of the sand fly vector, and a non-motile amastigote stage that populates that phagolysosome of mammalian macrophages . Leishmania mexicana promastigotes are coated with a number of GPI-anchored proteins, and lipophosphoglycans (LPGs) and glycosylinositol phospholipids (so-called GIPLs). These three classes of molecules share a common GPI-like Man 1 1-4GlcNo1-6inositol-phospholipid structure while their further carbohydrate structures are different. 
The early GPI anchor precursors of Leishmania mexicana fall into two distinct classes containing 1-alkyl-2-acyl-glycerols with either a C18 alkyl chain, or very long alkyl chains (C24, C26). In the protein-linked GPIs, only C24/C26 alkyl GPIs are incorporated whereas GIPLs only contain C18 alkyls. In the total cellular pool of glycerylphosphorylinositol lipids the C18 alkyl species accounts for $20 \%$, the C24/C26 alkyl species for less than 1\% [35]. The exclusive use of a rare glycerylphosphorylinositol lipid for GPI anchoring however is not thought of as being due to a remodeling reaction but rather to predilection for C24/C26 alkyls amongst the enzymes making GPIs for protein anchoring [19, 35].

However, Leishmania mexicana remodels the fatty acids of its free GIPLs by operating the removal of a C18 fatty acid from the the sn-2 position and incorporation of myristate in its stead, myristoly-CoA being the donor substrate. This reaction is reminiscent of the GPI lipid remodeling in T. brucei and may also occur on the GPIs used to anchor proteins [35].

\subsection{GPI remodeling in mammalian organisms}

A number of human and mammalian GPI anchors contain 1-alkyl-2-acyl-glycerol or 1-alkylglycerol as lipid moieties, while other mammalian GPI proteins contain regular DAG anchors [16]. 1-alkyl-2-acyl-sn-glycero-3-phosphorylinositol is present as free lipid in certain cell lines [36] and the alkyl-acyl-glycerol moieties are present already in the early steps of GPI biosynthesis in the same proportions as they are found in the mature GPI proteins [37-39], even when the level of 1alkyl-2-acyl-sn-glycero-3-phosphorylinositolphosphorylinositol in the cell is very low. This suggests (but does not prove) that the GPI-GlcNAc transferase achieving step 1 (Fig. 2) has a preference for 1-alkyl-2-acyl-sn-glycero-3-phosphorylinositol. Thus, as in L. mexicana, there is no need to invoke lipid remodeling to explain the presence of the very uncommon 1-alkyl-2-acyl-snglycero-3-phosphorylinositols in mammalian GPI anchors.

Interestingly, a recent report shows that mammalian GPI anchors seem to be remodeled and undergo a deacylation - reacylation cycle in the Golgi, mediated by PGAP2, which is believed to 
act as an acyltransferase [40]. This suggests that GPI lipid remodeling of protein-bound anchors may be a common event also in mammalian cells but the benefit of this is presently unclear.

\subsection{GPI remodeling in Saccharomyces cerevisiae}

The lipid moieties of mature GPI-anchors of Saccharomyces cerevisiae usually do not contain the DAG present on the GPI lipid at the stage when it is transferred by the transamidase to GPI proteins and which probably contains the C16 and C18 fatty acids found in yeast PI (Fig. 1)[4144]. Most mature GPI proteins of yeast contain a ceramide moiety, whereas a minor fraction contains a modified DAG containing C26:0 in sn-2 [10, 42](Fig. 1B). It has to be noted that ceramides of S.cerevisiae almost exclusively contain C26 fatty acids. Thus, all mature GPI proteins of yeast contain large lipid moieties with C26 or hydroxylated C26 fatty acids, either in the form of a ceramide or a special DAG and these lipids are introduced by remodeling enzymes that replace the primary lipid moiety of the anchor (Fig. 3A). Ceramides are also found in the GPI anchors in certain plants, (e.g. pears), Trypanosoma cruzi, Paramecium primaurelia, Aspergillus niger, Aspergillus fumigatus and Dictyostelium [16], sometimes as the sole anchor lipid [31]. The yeast remodeling activity introducing ceramide (ceramide remodelase) can be monitored by metabolic labeling experiments using tritiated DHS $\left(\left[{ }^{3} \mathrm{H}\right] \mathrm{DHS}\right)$ [43]. When given to cells, this tracer is rapidly taken up and is incorporated into all sphingolipids as well as ceramide-containing GPI proteins. By metabolic labeling experiments in different secretion mutants with one finds that ceramide remodelase activity is present in both, the ER and beyond, i.e. the Golgi or the plasma membrane [43]. Ceramides with hydroxylated fatty acids (C26-OH) are only introduced in the Golgi while other remodeling reactions can occur in the ER. Even on mature proteins, the ceramide moieties continue to be turned over (Fig. 3A)[43]. The identity of the enzymes introducing ceramide is presently not known, but it is clear that this activity is different from Aur1p, the IPC synthase, which may perform a formally similar reaction. Indeed, the inhibition of Aur1p by aureobasidin A completely blocks IPC biosynthesis, but does not affect GPI remodeling, showing 
that Aur1p does not mediate remodeling [45]. Moreover, short chain ceramides are ultilized by Aur1p but not by the ceramide remodelase. Many yeast strains, which have deletions in genes showing some homology with phospholipases A, B or C, with enzymes synthesizing or utilizing ceramides, with acyltransferases, or with other proteins binding acyl-CoA were tested for deficiences in GPI remodeling. Part of this effort has been published on the web by the EUROFAN2 lipid node at http://mips.gsf.de/proj/eurofan/eurofan_2/n7/conzelmann.html. Although some strains showed abnormalities in their sphingolipid profile, none of them was deficient in GPI remodeling. Incidentally it was found that gpi7 $\Delta$ cells are deficient in Golgi remodeling [46]. It may be that the Golgi remodelase introducing ceramide needs GPI protein substrates carrying a phosphorylethanolamine on Man2 of their anchor. Renewed screening of deletion strains has recently identified GUP1 as being essential for the synthesis of the C26:0containing diacylglycerol anchors (Fig. 3B)(Bosson et al., in press). Gup1p is an ER membrane protein with multiple membrane spanning domains harboring a motif that is characteristic of membrane bound $\underline{\text { O}}$-ạcyl-transferases (MBOAT motif). Present evidence suggests that Gup1p may act as an sn-2 specific acyltransferase in the remodeling of GPI anchors.

\section{REFERENCES}

1. Caro, L.H., Tettelin, H., Vossen, J.H., Ram, A.F., van den Ende, H. and Klis, F.M. (1997) Yeast 13, 1477-1489

2. De Groot, P.W., Hellingwerf, K.J. and Klis, F.M. (2003) Yeast 20, 781-796

3. Hamada, K., Fukuchi, S., Arisawa, M., Baba, M. and Kitada, K. (1998) Mol Gen Genet 258, 5359

4. Eisenhaber, B., Schneider, G., Wildpaner, M. and Eisenhaber, F. (2004) J Mol Biol 337, 243253

5. Leidich, S.D., Drapp, D.A. and Orlean, P. (1994) J Biol Chem 269, 10193-10196 
6. Caras, I.W., Weddell, G.N., Davitz, M.A., Nussenzweig, V. and Martin, D.W.J. (1987) Science 238, $1280-1283$

7. Moran, P. and Caras, I.W. (1991) J Cell Biol 115, 1595-1600

8. Gerber, L.D., Kodukula, K. and Udenfriend, S. (1992) J Biol Chem 267, 12168-12173

9. Nuoffer, C., Horvath, A. and Riezman, H. (1993) J Biol Chem 268, 10558-10563

10. Fankhauser, C., Homans, S.W., Thomas-Oates, J.E., McConville, M.J., Desponds, C., Conzelmann, A. and Ferguson, M.A. (1993) J Biol Chem 268, 26365-26374

11. Imhof, I., Flury, I., Vionnet, C., Roubaty, C., Egger, D. and Conzelmann, A. (2004) J Biol Chem 279, 19614-19627

12. Ferguson, M.A., Homans, S.W., Dwek, R.A. and Rademacher, T.W. (1988) Science 239, 753759

13. Homans, S.W., Ferguson, M.A., Dwek, R.A., Rademacher, T.W., Anand, R. and Williams, A.F. (1988) Nature 333, 269-272

14. Ferguson, M.A., Brimacombe, J.S., Brown, J.R., Crossman, A., Dix, A., Field, R.A., Guther, M.L., Milne, K.G., Sharma, D.K. and Smith, T.K. (1999) Biochim Biophys Acta 1455, 327340

15. Ferguson, M.A. (1999) J Cell Sci 112, 2799-2809

16. Ferguson MAJ, Kinoshita T, Hart GW. Glycophospholipid anchors. In: Varki A, Bertozzi C, Cummings R, Etzler M, Esko J, Freeze H, Hart G, Stanley P, editors. 2nd edition. in press.

17. Eisenhaber, B., Maurer-Stroh, S., Novatchkova, M., Schneider, G. and Eisenhaber, F. (2003) Bioessays 25, 367-385

18. Ikezawa, H. (2002) Biol Pharm Bull 25, 409-417

19. McConville, M.J. and Menon, A.K. (2000) Mol Membr Biol 17, 1-16

20. Ralton, J.E., Mullin, K.A. and McConville, M.J. (2002) Biochem J 363, 365-375

21. Baumann, N.A., Vidugiriene, J., Machamer, C.E. and Menon, A.K. (2000) J Biol Chem 275, 7378-7389

22. Singh, N., Liang, L.N., Tykocinski, M.L. and Tartakoff, A.M. (1996) J Biol Chem 271, 1287912884

23. Puoti, A. and Conzelmann, A. (1992) J Biol Chem 267, 22673-22680 
24. Masterson, W.J., Raper, J., Doering, T.L., Hart, G.W. and Englund, P.T. (1990) Cell 62, 73-80

25. Buxbaum, L.U., Raper, J., Opperdoes, F.R. and Englund, P.T. (1994) J Biol Chem 269, 3021230220

26. Buxbaum, L.U., Milne, K.G., Werbovetz, K.A. and Englund, P.T. (1996) Proc Natl Acad Sci U S A 93, 1178-1183

27. Doering, T.L., Pessin, M.S., Hart, G.W., Raben, D.M. and Englund, P.T. (1994) Biochem J 299, 741-746

28. Morita, Y.S., Acosta-Serrano, A., Buxbaum, L.U. and Englund, P.T. (2000) J Biol Chem 275, $14147-14154$

29. Milne, K.G. and Ferguson, M.A. (2000) J Biol Chem 275, 12503-12508

30. Bertello, L.E., Alves, M.J., Colli, W. and de Lederkremer, R.M. (2004) Mol Biochem Parasitol 133, $71-80$

31. Fontaine, T., Magnin, T., Melhert, A., Lamont, D., Latge, J.P. and Ferguson, M.A. (2003) Glycobiology 13, 169-177

32. Costachel, C., Coddeville, B., Latge, J.P. and Fontaine, T. (2005) J Biol Chem 280, 3983539842

33. Levery, S.B., Toledo, M.S., Straus, A.H. and Takahashi, H.K. (2001) Rapid Commun Mass Spectrom 15, 2240-2258

34. Fontaine, T., Smith, T.K., Crossman, A., Brimacombe, J.S., Latge, J.P. and Ferguson, M.A. (2004) Biochemistry 43, 15267-15275

35. Ralton, J.E. and McConville, M.J. (1998) J Biol Chem 273, 4245-4257

36. Lee, T.C., Malone, B., Buell, A.B. and Blank, M.L. (1991) Biochem Biophys Res Commun 175, 673-678

37. Puoti, A., Desponds, C., Fankhauser, C. and Conzelmann, A. (1991) J Biol Chem 266, 2105121059

38. Butikofer, P., Zollinger, M. and Brodbeck, U. (1992) Eur J Biochem 208, 677-683

39. Camp, L.A., Chauhan, P., Farrar, J.D. and Lehrman, M.A. (1993) J Biol Chem 268, 6721-6728

40. Tashima, Y., Taguchi, R., Murata, C., Ashida, H., Kinoshita, T. and Maeda, Y. (2006) Mol

Biol Cell 17, 1410-1420 
41. Conzelmann, A., Puoti, A., Lester, R.L. and Desponds, C. (1992) EMBO J 11, 457-466

42. Sipos, G., Reggiori, F., Vionnet, C. and Conzelmann, A. (1997) EMBO J 16, 3494-3505

43. Reggiori, F., Canivenc-Gansel, E. and Conzelmann, A. (1997) EMBO J 16, 3506-3518

44. Schneiter, R., Brugger, B., Amann, C.M., Prestwich, G.D., Epand, R.F., Zellnig, G., Wieland, F.T. and Epand, R.M. (2004) Biochem J 381, 941-949

45. Reggiori, F. and Conzelmann, A. (1998) J Biol Chem 273, 30550-30559

46. Benachour, A., Sipos, G., Flury, I., Reggiori, F., Canivenc-Gansel, E., Vionnet, C., Conzelmann, A. and Benghezal, M. (1999) J Biol Chem 274, 15251-15261

47. Sipos, G., Puoti, A. and Conzelmann, A. (1995) J Biol Chem 270, 19709-19715

48. Canivenc-Gansel, E., Imhof, I., Reggiori, F., Burda, P., Conzelmann, A. and Benachour, A. (1998) Glycobiology 8, 761-770

49. Sutterlin, C., Escribano, M.V., Gerold, P., Maeda, Y., Mazon, M.J., Kinoshita, T., Schwarz, R.T. and Riezman, H. (1998) Biochem J 332, 153-159

50. Taron, C.H., Wiedman, J.M., Grimme, S.J. and Orlean, P. (2000) Mol Biol Cell 11, 1611-1630

51. Grimme, S.J., Westfall, B.A., Wiedman, J.M., Taron, C.H. and Orlean, P. (2001) J Biol Chem 276, 27731-27739 


\section{ACKNOWLEDGEMENTS}

Thanks to Mike Ferguson for share out a review in press. This work was supported by a grant from the Swiss National Foundation to A.C.

\section{FIGURE LEGENDS}

Figure 1. Structure of GPI anchors of yeast. A, the average structure of the yeast GPI anchor as elaborated from the total pool of GPI anchors $[10,11]$. The carbohydrate core structure, which is conserved throughout the eukaryotic kingdom is shown in bold characters. The box contains CP2, the most mature yeast GPI lipid accumulating in GPI transamidase mutants; CP2 contains 4 mannoses (Man1 to Man 4) and phosphorylethanolamine (EtNH $2-\mathrm{P})$ substituents on Man1, Man2 and Man3. A $5^{\text {th }}$ mannose (Man5) can be added to GPI proteins while they transit through the Golgi apparatus, whereby Man5 is added either in $\alpha 1-2$ or in $\alpha 1-3$ linkage [10, 47]. Although present on $\mathrm{CP} 2$, it is not certain that the $\mathrm{EtNH}_{2}-\mathrm{P}$ on Man2 is present on GPI anchors of proteins [11]. The palmitic acid on the inositol (Ins) is present on all but the earliest free GPI lipids but it is removed as soon as the GPI lipid is attached to a protein (see step 12 in Fig. 2). B, the two alternative lipid moieties found on mature GPI proteins are shown. In both lipid moieties, part of the C26 fatty acids are $\alpha$-hydroxylated [10]. In CP2, x and y are probably 14 and 15, on mature protein anchors they are 14 and 23, respectively.

Figure 2. The main pathway of GPI biosynthesis in yeast. Since no GPI intermediates are detectable in wild type cells, a likely order of 12 consecutive reactions is proposed based on 
structures of GPIs accumulating in well characterized gpi mutants [46, 48-51]. CP2 is the most polar lipid accumulating in gpi8 mutants, M4 accumulates in gpi7 $\Delta$ cells and is also transferred to GPI proteins [46].

Figure 3. Proposed remodeling reactions. A, the various lipids found on mature GPI anchored proteins are depicted together with the presumed base-sensitive PI (top) that is present on CP2 (Fig. 1) and is transferred to proteins by the transamidase. A kinetic analysis of anchor lipids appearing after various times of metabolic labeling of cells with $\left[{ }^{3} \mathrm{H}\right]$ inositol suggests that the anchors with a C16/C18 DAG are first transformed into anchors with C16/C26 fatty acids and only thereafter ainto ceramide (Cer) containing anchors as indicated by the arrows 1 and 2 on the left [42]. Recent data confirm this notion (Bosson et al., in press). The arrows to the right are to show that ceramides continue to be exchaged for ceramides even on mature GPI proteins [43]. B, recent data indicate that arrow 1 in panel A consists of 2 reactions, one of which depends on GUP1. Other data however suggest that a direct transition from anchors with a C16/C18 containing DAG to a ceramide anchor may also take place (thin arrows; panel A, arrow 3)(Bosson et al., in press). 


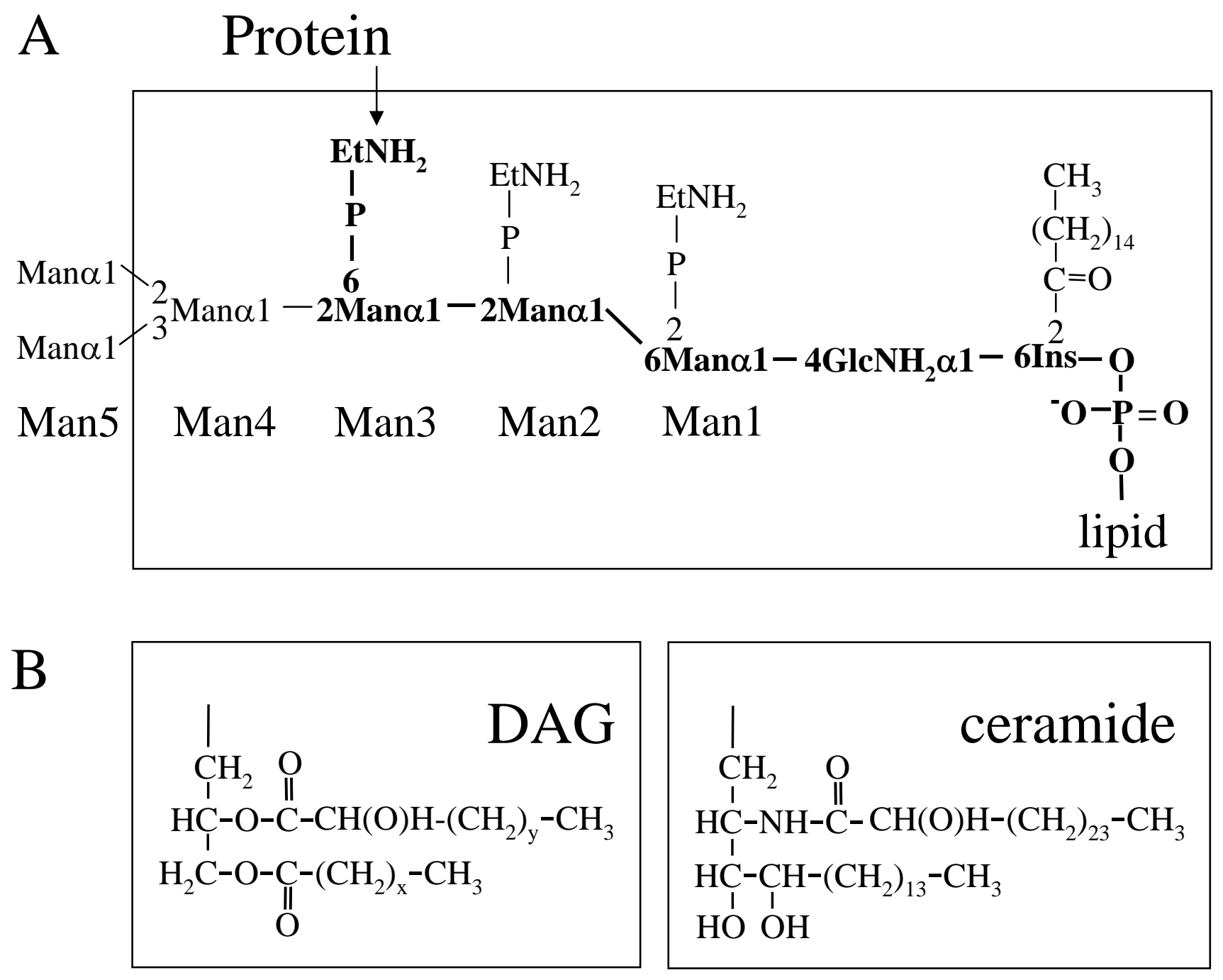

Bosson and Conzelmann, Figure 1 


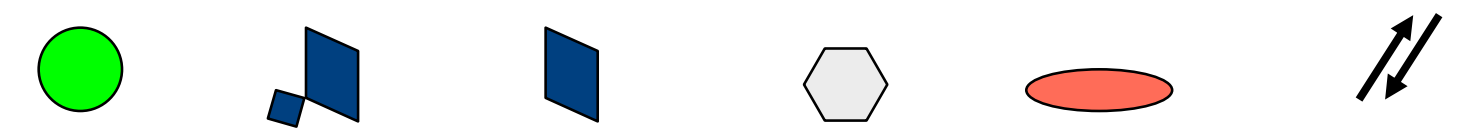
inositol GlcNAc GlcNH $_{2}$ mannose EtN-P Flip

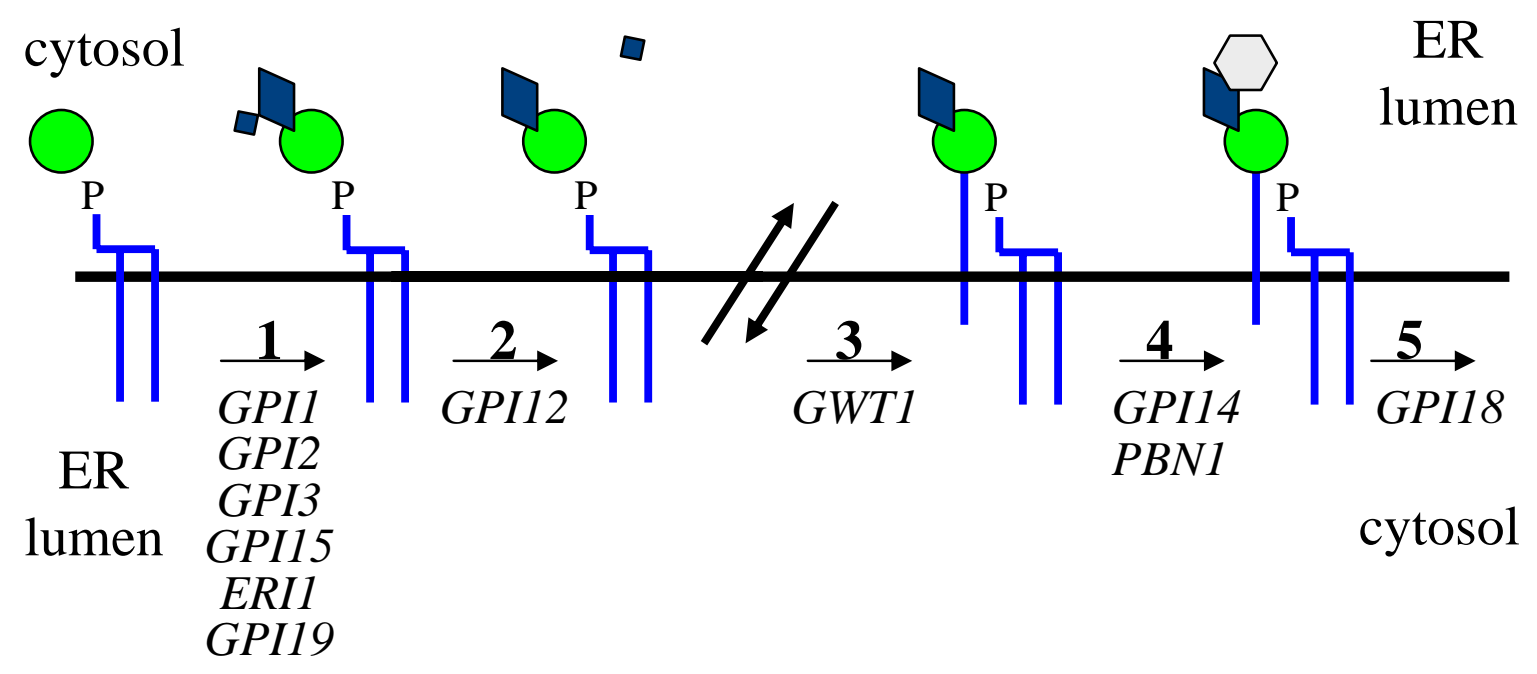

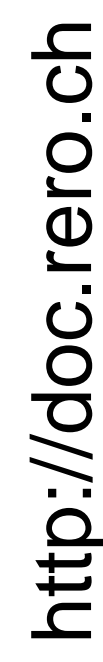
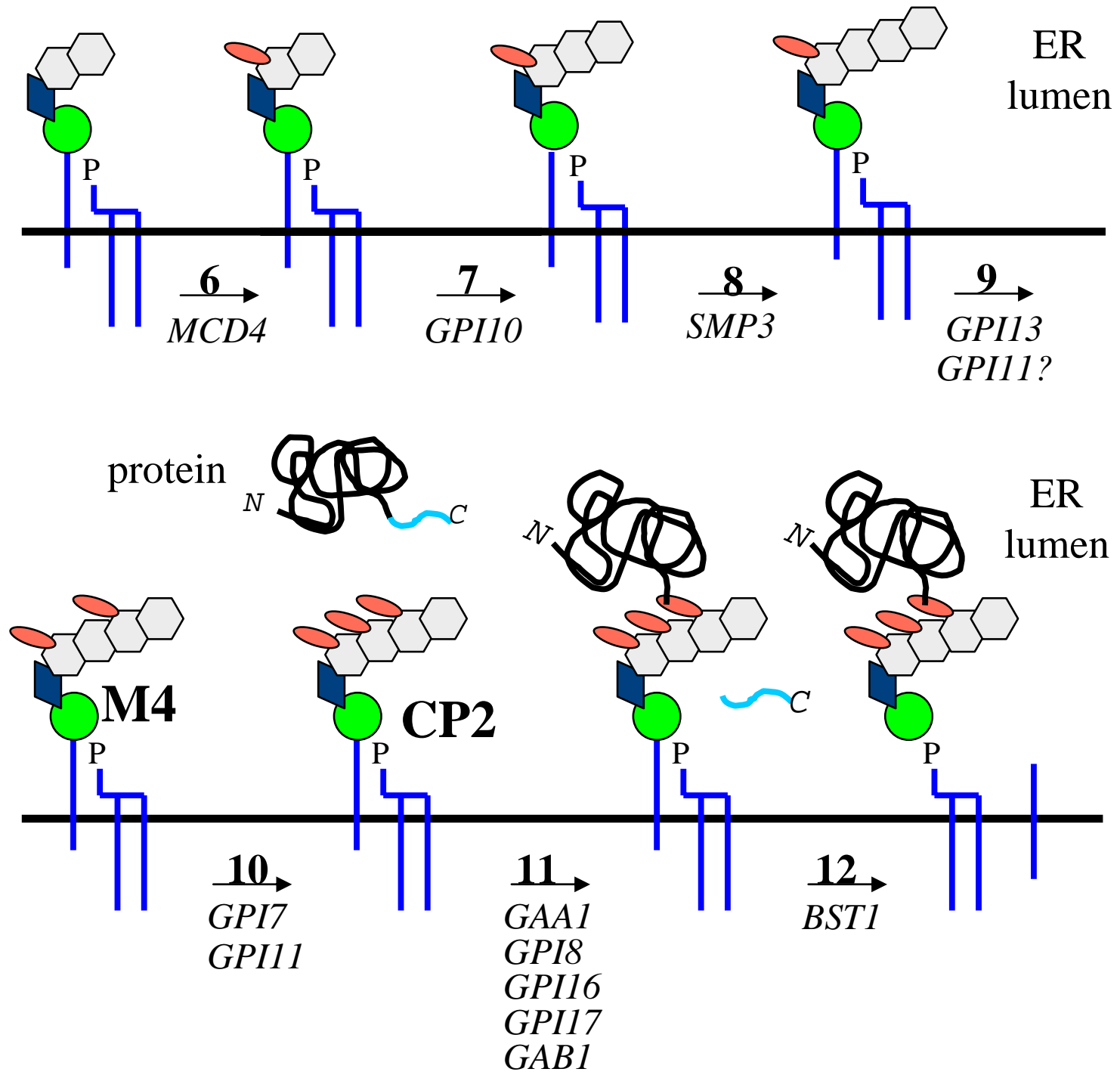

Bosson and Conzelmann, Figure 2 

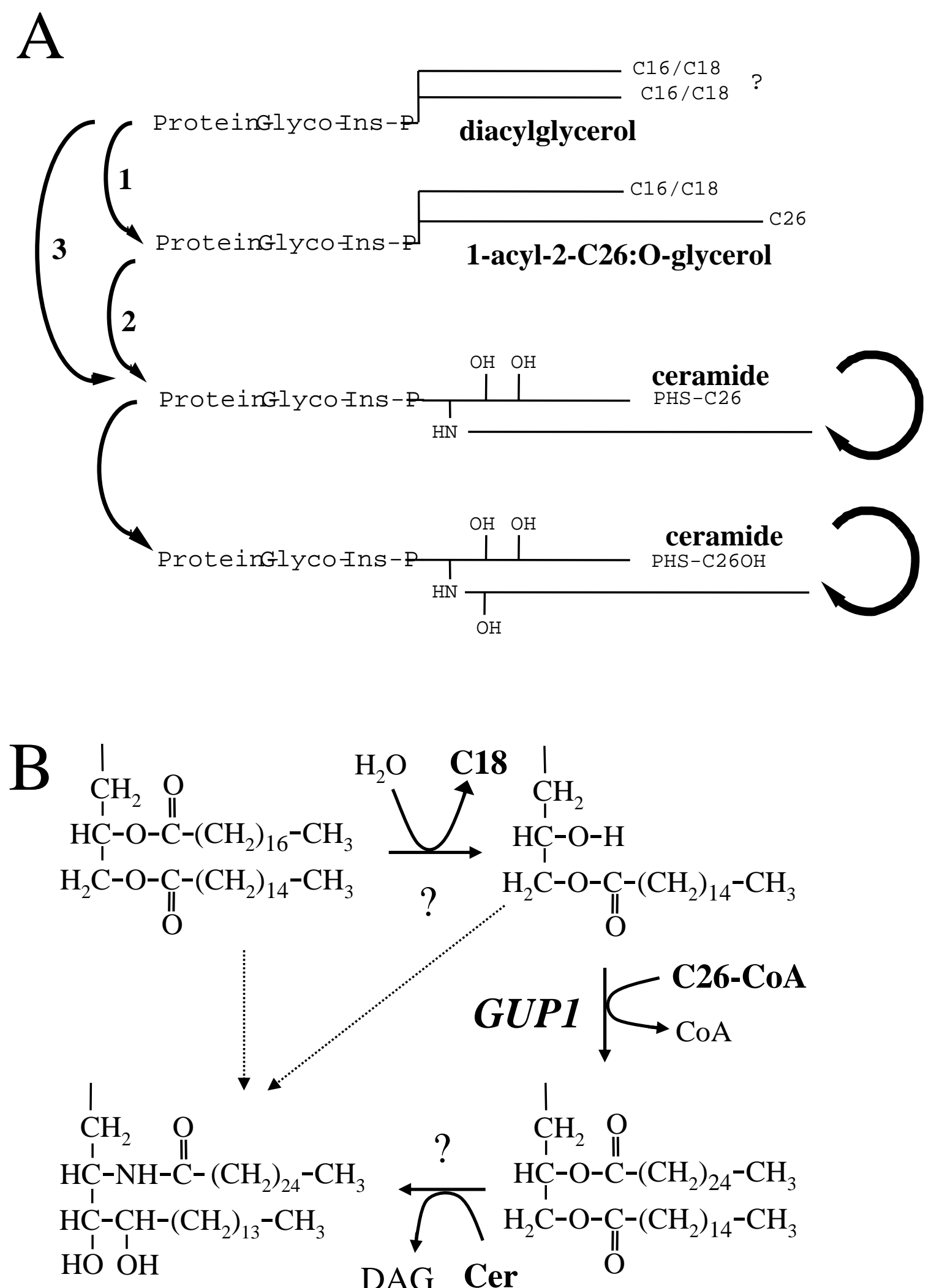

Bosson and Conzelmann, Figure 3 